\title{
Acute viral infection results in a PD-1-dependent loss of anti-tumor CD8+ T cell responses: implications for tumor immunotherapy
}

\author{
Frederick Kohlhapp ${ }^{1}$, Erica Huelsmann², Joseph Broucek², Jason Schenkel ${ }^{3}$, Howard Kaufman ${ }^{1}$, Andrew Zloza ${ }^{1 *}$ \\ From 30th Annual Meeting and Associated Programs of the Society for Immunotherapy of Cancer (SITC 2015) \\ National Harbor, MD, USA. 4-8 November 2015
}

\begin{abstract}
Introduction
Oncolytic viruses are gaining acceptance as a method of tumor immunotherapy, and some studies have reported improved anti-tumor $\mathrm{T}$ cell responses after acute pyretic non-oncolytic infections. Therefore, we hypothesized that viral infections may aid in the immunotherapy of cancer.
\end{abstract}

\section{Methods}

To test this hypothesis, we used the A/H1N1/PR8 mouse model of influenza infection $(10,000 \mathrm{pfu}$, via intranasal administration) prior to or after challenge with B16 melanoma (12,000 - 120,000 cells via intradermal injection in the right flank). Kaplan-Meier curves were used to present animal survival and tumor incidence data and log rank test was used to compare such curves. All statistical analyses were performed using Prism software (v4.0, GraphPad software), and a p value of less than 0.05 was considered to denote statistically significant differences among compared variables.

\section{Results}

Contrary to our hypothesis, we observed that acute influenza infection of the lung promotes distant melanoma growth in the dermis of the flank and results in up to $50 \%$ decrease in host survival $(\mathrm{P}<0.001)$. We observed similar findings across a series of different infection and tumor models. In experiments designed to determine whether infection could lead to the emergence of cancer that is otherwise controlled by the immune system, we challenged mice with serial cell numbers of B16 $(12,000$ and 1,200) at which tumor incidence is reduced (to $60 \%$ and $0 \%$, respectively). Here,

${ }^{1}$ Rutgers CINJ, New Brunswick, NJ, USA

Full list of author information is available at the end of the article influenza infection significantly increased tumor incidence for both 12,000 and 1,200 B16 cell challenges to $100 \%$ ( $\mathrm{P}<0.01$ and $\mathrm{P}<0.001$, respectively). Further, using a tumor transplant model we demonstrated that anti-melanoma CD8 $+\mathrm{T}$ cells (defined by a congenic marker and/or tetramer) in the context of influenza infection are shunted to the lungs $(\mathrm{P}<0.01$ vs noninfected mice), where they express high levels of the activation and exhaustion receptor, PD-1 ( $<<0.01)$. Immunotherapy with PD-1 blockade reverses this loss of anti-tumor $\mathrm{CD} 8+\mathrm{T}$ cells from the tumor and decreases viral infection-induced tumor growth.

\section{Conclusions}

Our findings define a previously unrecognized mechanism of immune suppression in cancer-bearing hosts, namely acute non-oncogenic viral infection. This finding may be important because it raises concerns over patient exposure to acute viral illness, vaccination, and treatment with viral vectors in tumor immunotherapy; and it highlights an unexpected mechanism of PD-1 blockade in the treatment of cancer.

\footnotetext{
Authors' details

${ }^{1}$ Rutgers CINJ, New Brunswick, NJ, USA. ${ }^{2}$ Rush University, Chicago, IL, USA.

${ }^{3}$ University of Minnesota, Minneapolis, MN, USA.
}

Published: 4 November 2015

doi:10.1186/2051-1426-3-S2-P281

Cite this article as: Kohlhapp et al:: Acute viral infection results in a

PD-1-dependent loss of anti-tumor CD8+ T cell responses: implications

for tumor immunotherapy. Journal for ImmunoTherapy of Cancer 20153

(Suppl 2):P281. 